\title{
Caracterización de suelos bajo bosques de Nothofagus betuloides (Mirb) Blume, en Tierra del Fuego, Chile
}

\author{
Characterization of soils of Nothofagus betuloides (Mirb) Blume forests, in Tierra del \\ Fuego, Chile
}

VÍCTOR GERDING \& OSCAR THIERS

Instituto de Silvicultura, Universidad Austral de Chile, Casilla 567, Valdivia, Chile; e-mail:vgerding@uach.cl

\begin{abstract}
RESUMEN

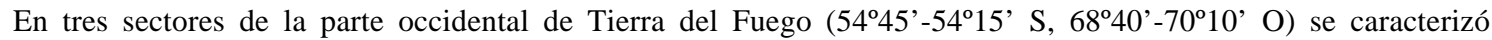
morfológica, física y químicamente los suelos bajo bosques de coigüe de Magallanes (Nothofagus betuloides (Mirb) Blume) y sus especies asociadas lenga (Nothofagus pumilio (P. et E.) Krasser) y canelo (Drimys winteri Forst.). Se describieron 85 perfiles de suelo y aproximadamente 250 observaciones con bastón pedológico. Los suelos bajo coigüe de Magallanes eran jóvenes, muy influidos por la actividad volcánica, topografía y clima. Se observó la presencia de los órdenes Spodosol e Iceptisol, principalmente. En general los suelos eran delgados a muy delgados (mayoritariamente entre 10 y $40 \mathrm{~cm}$ ), con un alto volumen de esqueleto, textura franca, estructura masiva a granular, capacidad de agua aprovechable baja y drenaje externo e interno moderado a lento. Además, presentan una alta acidez (pH 4-5,5), una baja oferta nutricional y muy altos niveles de saturación de aluminio (promedio > $60 \%$ ). El mantillo (Oe/Oa) presentó condiciones adecuadas para el desarrollo radicular. Existe comúnmente un horizonte blanco (frecuentemente 10 YR 5/1-2) inmediatamente debajo del mantillo, con textura franco limosa y estructura masiva, con arraigamiento muy bajo. Se plantea como hipótesis que este horizonte se originó por depósitos de cenizas volcánicas y procesos de podzolización. Se concluye que la fertilidad de estos suelos es baja debido a limitantes físicas (dificultades de arraigamiento) y químicas (acidez, toxicidad de aluminio, baja oferta de bases).
\end{abstract}

Palabras clave: fertilidad de suelos, Nothofagus betuloides, Tierra del Fuego.

\begin{abstract}
Soils under coigüe de Magallanes (Nothofagus betuloides (Mirb) Blume) forests, located in the oriental part of Tierra del Fuego $\left(54^{\circ} 45^{\prime}-54^{\circ} 15^{\prime} \mathrm{S}, 68^{\circ} 40^{\prime}-70^{\circ} 10^{\prime} \mathrm{W}\right)$, were characterized morphologically, physically and chemically. Associated tree species were lenga (Nothofagus pumilio (P. et E.) Krasser) and canelo (Drimys winterii Forst.). A total of 85 soil profiles and approximately 250 soil cores were described. The soils in the $N$. betuloides forests were young, heavily influenced by the volcanic activity, topography and climate. The presence of the orders Spodosol and Iceptisol was recorded. Soils range from thin to very thin, with a high skeleton volume, loamy texture, structure ranging from massive to granular, with a low water retention capacity, and a moderate to slow drainage. They present high acidity ( $\mathrm{pH}$ 4-4.5), low nutrient concentration and high levels of aluminum. The litter layer (Oe/Oa) presents adequate root development. There was generally a white horizon (frequently 10 YR 5/1-2) immediately below the litter, with a loamy texture, massive structure, and low rooting levels. The origin of this horizon is hypothesized to be related with pedogenic factors on volcanic ash deposits. It is concluded that the fertility of forest soils is low, mainly due to physical and chemical characteristics.
\end{abstract}

Key words: soil fertility, Nothofagus betuloides, Tierra del Fuego.

\section{INTRODUCCIÓN}

La región de Tierra del Fuego, en su parte occidental, presenta extensas superficies boscosas (CONAF et al. 1997). Entre las principales especies forestales destaca Nothofagus betuloides, coigüe de Magallanes, que participa en los tipos forestales lenga y coigüe de Magallanes (Donoso 1981). La relación entre estos bosques y los sue- los donde se desarrollan es un aspecto poco conocido, existiendo escasos antecedentes sobre los suelos de la región (Díaz et al. 1960, Pisano 1977). Sadzawka et al. (1995) describieron algunos suelos desde el punto de vista químico. Para la porción argentina de Tierra del Fuego, INTAORSTOM (1991) tratan aspectos de la pedogénesis. En la región noroccidental de Tierra del Fuego se ha observado ${ }^{1}$ : (a) mayor creci- 
miento de los bosques de $N$. betuloides cuando éstos se desarrollan en suelos más profundos, (b) que en suelos con restricciones moderadas de drenaje domina $N$. betuloides por sobre N. pumilio y (c) que a elevaciones por sobre los $300 \mathrm{~m}$, el suelo disminuye su profundidad.

La pedogénesis presenta todavía muchas incógnitas y es posible encontrar descripciones de suelos podzólicos, aunque algunos autores no son tan precisos en esta caracterización, por lo que hay controversia (Thiers 1997). Por otra parte, las características químicas y morfológicas de los suelos son una respuesta a condiciones del ambiente e inducen a cambios y posibilidades de establecimiento y crecimiento de la vegetación, que a su vez afectan los suelos.

Este trabajo contribuye al conocimiento de los suelos de Tierra del Fuego y su relación con los bosques regionales. Los objetivos específicos son: (1) caracterizar suelos asociados principalmente a bosques de $N$. betuloides, con énfasis en su morfología y aspectos químico - nutritivos; y (2) evaluar la fertilidad de los suelos, detectando sus principales factores limitantes para el desarrollo de la vegetación.

\section{MATERIALES Y MÉTODOS}

El estudio se realizó dentro del patrimonio de la empresa Forestal Trillium Limitada, en la Isla de Tierra del Fuego (Fig. 1); la superficie aproximada de la propiedad es de 300.000 ha, dividida en tres sectores: Río Bueno, Río Cóndor y Vicuña. De estos sectores se estudiaron con mayor énfasis los dos primeros, por ser los que presentan mayor cobertura de bosques de $N$. betuloides (Donoso et al. 1995², Thiers 1997). Del sector de Vicuña se describen suelos de algunos sitios con bosques de lenga (Nothofagus pumilio). La toma de datos en terreno se realizó durante el verano de 19941995.

${ }^{1}$ GERDING V, H HUBER \& A FAESCH (1995)

Reconocimiento de suelos. Forestal Trillium Ltda., Proyecto Río Cóndor, Informe del subproyecto 9402. Facultad de Ciencias Forestales, Universidad Austral de Chile, Valdivia, Chile. 76 pp.

${ }^{2}$ DONOSO C, P DONOSO, M GONZÁLEZ \& C MAUREIRA (1995) Tipos forestales y estructura. Forestal Trillium Ltda., Proyecto Río Cóndor. Universidad Austral de Chile, Facultad de Ciencias Forestales, Valdivia, Chile. 108 pp.

\section{Clima}

El clima de la zona ha sido descrito por Díaz et al.(1960), Fuenzalida (1965), IREN (1967) y Pisano (1977). De acuerdo con la clasificación de Köppen, el sector de Río Bueno presenta un clima transandino con degeneración esteparia (Dfk'c), con una precipitación anual cercana a los $500 \mathrm{~mm}$ y una temperatura media anual de alrededor de 5 ${ }^{\circ} \mathrm{C}$. En el sector de Río Cóndor el clima es templado frío con gran humedad (Cfk'c), con una precipitación anual cercana a $1.000 \mathrm{~mm}$ y una temperatura media anual levemente superior a la del sector Río Bueno. La precipitación se distribuye uniformemente a través del año. En toda la zona los vientos dominantes son del sudoeste y oeste, con velocidades que alcanzan $150 \mathrm{~km} \mathrm{~h}^{-1}$, representando un importante agente erosivo y de desecamiento de los suelos, principalmente en primavera y verano.

\section{Geomorfología y geología}

El estudio se concentró en la franja denominada precordillera oriental o subandina oriental. Es una zona mesetiforme atravesada por cordones montañosos de baja altura, cuyas cumbres rara vez superan los 1.000 a $1.200 \mathrm{~m}$. Fueron fuertemente modificados por efecto de los hielos cuaternarios y fenómenos postglaciales, entre los cuales se destaca el labrado de amplios valles. Estos valles están rellenos con depósitos glaciales, glacifluviales, fluviales y lacustres (Pisano 1977, INTA-ORSTON 1991, Dollenz 1995).

El sustrato geológico de esta región está formado por depósitos marinos de distintas edades (Pisano 1977). Los más jóvenes (aproximadamente entre 45 y 65 millones de años) se encuentran en Río Bueno, sector ubicado al norte de la zona de estudio; el sector de Río Cóndor presenta depósitos de una mayor edad (aproximadamente 100 millones de años). Además se han determinado extensos depósitos de cenizas volcánicas (Díaz et al.1960, INTA-ORSTON 1991, Arroyo et al. $\left.1996^{3}\right)$.

\footnotetext{
${ }^{3}$ ARROYO M, C DONOSO, R MURÚA, E PISANO, R SCHLATTER \& I SEREY (1996)

Toward an ecologically sustainable forestry project: concepts, analysis and recommendations. Departamento de Investigación y Desarrollo, Universidad de Chile, Santiago, Chile. 253 pp.
} 




\section{Área del proyecto Trillium}

(1): Punta Arenas

(3): Tierra del Fuego

(5): Sector Rio Bueno

(7): Puerto Arturo

(9): Sector Vicuña

(11): Seno Almirantazgo
(2): Estrecho de Magallanes

(4): Bahia Inútil

(6): Sector Rio Cóndor

(8): Sector Río Paralelo

(10): Lago Blanco

(12): Lago Fagnano

Fig. 1: Ubicación de los sectores de estudio (modificado de Arroyo et al.1996).

Location of the study areas (modified from Arroyo et al.1996).

\section{Topografía}

En el sector de Río Bueno se estudiaron sitios en terrenos bajos planos, intermedios planos e intermedios ondulados a quebrados (105 a $340 \mathrm{~m}$ de altitud). El paisaje está dominado por planos y lomajes de laderas rectas a convexas con pendientes entre 1 y $65 \%$. La exposición noroeste fue la más comúnmente evaluada. El drenaje externo del suelo es principalmente lento, con escasa variación en todo el sector. Los sitios muestreados en Vicuña coinciden con las características topográficas de Río Bueno.

En Río Cóndor se observaron sitios en terrenos bajos planos, terrenos de elevación intermedia planos y ondulados a quebrados, y rara vez terrenos altos (10-435 m). Las posiciones fisiográficas donde se observaron las diferentes asociaciones de $N$. betuloides fueron lomas y cerros, y en menor proporción planos y terrazas (de río y mar). Se analizaron sitios en diversas ubicaciones en el relieve: planos, laderas (altas, medias, bajas) y terrazas (de río y de mar). Las formas cóncavas fueron las más comunes en las distintas laderas con pendientes de 0 a $55 \%$, y exposición NO-NE. El drenaje externo era mayoritariamente moderado, aumentando con el incremento de la pendiente.

Características de los bosques: Río Bueno

Los bosques relacionados con este trabajo son los siguientes:

Bosques de coigüe de Magallanes-lenga $(N$. betuloides, $N$. pumilio): es la formación más im- 
portante del sector, se ubican en la parte central de las masas boscosas que se encuentran entre las turberas. La densidad del bosque varía entre 400 y 500 árboles ha-1 y el área basal es mayor a $80 \mathrm{~m}^{2}$ $\mathrm{ha}^{-1}$. Nothofagus betuloides alcanza alturas entre los 18 y $28 \mathrm{~m}$.

Bosques de coigüe de Magallanes puro ( $N$. betuloides): ubicados frecuentemente alrededor de las turberas. Son bosques multietáneos. La densidad fluctúa alrededor de 1.200 árboles ha ${ }^{-1} \mathrm{y}$ el área basal cercana a $100 \mathrm{~m}^{2} \mathrm{ha}^{-1}$. La altura de los árboles de $N$. betuloides alcanza los $30 \mathrm{~m}$.

Bosques de coigüe de Magallanes-canelo-ñirreleñadura ( $N$. betuloides, Drymis winteri, $N$. antarctica, Maytenus magelanica): propios de las áreas costeras. La densidad del bosque varía entre 1.200 y 1.500 árboles ha-1 y su área basal entre 50 y $70 \mathrm{~m}^{2} \mathrm{ha}^{-1}$. Las alturas que alcanza $N$. betuloides son cercanas a los $30 \mathrm{~m}$, mientras $D$. winteri no supera los $15 \mathrm{~m}$.

\section{Características de los bosques: Río Cóndor}

En este sector se observó mayor diversidad de especies y estructuras en los bosques.

Bosques de coigüe de Magallanes puro: se ubican desde la costa hasta el interior del sector. Los distintos sitios contemplan variadas fases sucesionales del bosque, razón por la cual la densidad y el área basal varían entre 500 y 2.000 árboles ha-1 y entre 50 y $100 \mathrm{~m}^{2} \mathrm{ha}^{-1}$, respectivamente. En esta asociación boscosa la altura de $N$. betuloides alcanza a $30 \mathrm{~m}$.

Bosques de coigüe de Magallanes-lenga: se ubican frecuentemente hacia el interior del sector. La densidad varía entre 500 y 600 árboles ha ${ }^{1}$ y el área basal entre 70 y $90 \mathrm{~m}^{2} \mathrm{ha}^{-1}$. Nothofagus betuloides domina a N. pumilio en densidad, mientras que esta última especie presenta árboles de mayor diámetro $(40-50 \mathrm{~cm})$. Las alturas de $N$. betuloides llegan a $35 \mathrm{~m}$.

Bosque de coigüe de Magallanes-canelo: propio de las áreas costeras, donde $N$. betuloides domina en altura (10-15 m), mientras que canelo frecuentemente no supera los $10 \mathrm{~m}$. Algunos bosques corresponden a renovales de $N$. betuloides.

Bosques de coigüe de Magallanes-ñirre: corresponde sólo a un sitio cercano al límite arbóreo altitudinal. Aquí $N$. betuloides presenta una altura de $20 \mathrm{~m}$, mientras que $N$. antarctica un desarro11 o cercano a los $10 \mathrm{~m}$.

Bosques de coigüe de Magallanes-lenga-canelo: corresponde a un sitio en terrenos bajos, cerca del Río Cóndor. La densidad del bosque es cercana a los 500 árboles ha ${ }^{-1} \mathrm{y}$ el área basal entre los 50 y $70 \mathrm{~m}^{2} \mathrm{ha}^{-1}$. Las especies $N$. betuloides y $N$. pumilio alcanzan a $\operatorname{los} 20 \mathrm{~m}$, en tanto que $D$. winteri no supera los $12 \mathrm{~m}$.

Reconocimiento y caracterización química de los suelos

El reconocimiento de suelos se realizó tanto con muestreos puntuales como en transectos orientados según la variación topográfica y vegetacional (Schlatter et al. 1981) ${ }^{4}$. Los sitios de muestreo se seleccionaron de acuerdo con la variación del bosque, de tal manera de abarcar las principales unidades que se detectaron a través de fotografías aéreas y de reconocimiento en terreno. De la misma forma, se establecieron transectos con catenas para observar la variación del suelo asociada a la topografía. Mediante calicatas se describieron los perfiles de suelo asociados a bosques con N. betuloides: 28 en el sector Río Bueno, 56 en Río Cóndor y uno en Vicuña. Se complementó la descripción con aproximadamente 250 observaciones realizadas a través de un bastón pedológico.

En cada sitio de muestreo se realizó una descripción que consideró (a) el paisaje a través de la forma del terreno, posición fisiográfica, ubicación en el relieve, pendiente, elevación, exposición y drenaje externo; (b) la morfología del mantillo a través de sus horizontes orgánicos, espesor, material, estructura y arraigamiento; y (c) el perfil de suelo en que se observó la profundidad y límites de los horizontes, el contenido de raíces finas, la estructura, la consistencia, la textura al tacto, la pedregosidad, el color, el drenaje interno, el nivel freático, las profundidades fisiológica y de raíces, el grado de erosión y los factores limitantes de la fertilidad (Schlatter $1993)^{5}$. En esta descripción se consideró también la capacidad de agua aprovechable del suelo, que equivale al volumen de poros medios del suelo, para evaluar el régimen de agua; y el drenaje interno y textura para caracterizar el régimen de aire del suelo. En la Tabla 1 se sintetizan las

${ }^{4}$ SCHLATTER J, R GREZ \& V GERDING (1981) Manual para el reconocimiento de suelos. Facultad de Ciencias Forestales, Universidad Austral de Chile, Valdivia, Chile. 81 pp.

${ }^{5}$ SCHLATTER JE (1993) Fertilidad del suelo, conceptos y su aplicación en la actividad forestal. Charlas y Conferencias 8, Facultad de Ciencias Forestales, Universidad Austral de Chile, Valdivia, Chile. Pp. 76-86. 
TABLA 1

Definiciones de los horizontes del suelo

Definitions of soil horizons

\begin{tabular}{|c|c|}
\hline Horizonte & Definición \\
\hline Oi & $\begin{array}{l}\text { Horizonte del mantillo compuesto de desechos orgánicos con mínima descomposición, donde se pueden } \\
\text { reconocer los tejidos de los materiales. El contenido de fibras es }>40 \% \text { (en volumen) }\end{array}$ \\
\hline Oe & $\begin{array}{l}\text { Horizonte del mantillo compuesto de desechos orgánicos con moderada (intermedia) descomposición; } \\
\text { el contenido de fibras es de } 17-40 \% \text { (en volumen) }\end{array}$ \\
\hline $\mathrm{Oa}$ & $\begin{array}{l}\text { Horizonte del mantillo compuesto de desechos orgánicos con alta descomposición, donde no son } \\
\text { reconocibles los tejidos orgánicos originales; contenido de fibra }<17 \% \text { (en volumen) }\end{array}$ \\
\hline A & $\begin{array}{l}\text { Horizonte mineral formado en la superficie o bajo un horizonte } \mathrm{O} \text {, con acumulación de materia orgánica } \\
\text { humificada íntimamente mezclada con la fracción mineral }\end{array}$ \\
\hline $\mathrm{E}$ & $\begin{array}{l}\text { Horizonte mineral cuya principal característica es la pérdida por eluviación de arcilla silicatada, hierro } \\
\text { y/o aluminio, permaneciendo una concentración de limo y arena. Se forma cerca de la superficie, bajo } \\
\text { un horizonte A y encima de un B. Generalmente es de color más claro y de menor contenido de materia } \\
\text { orgánica que un horizonte A }\end{array}$ \\
\hline $\mathrm{B}$ & $\begin{array}{l}\text { Horizonte mineral formado bajo un } \mathrm{A}, \mathrm{E} \text { u O. Entre sus características más destacadas está la } \\
\text { acumulación de arcilla silicatada, hierro, aluminio, humus y otros compuestos }\end{array}$ \\
\hline Bhs & $\begin{array}{l}\text { Horizonte B con acumulación iluvial de materia orgánica humificada (h) y de sesquióxidos (s); es normal } \\
\text { encontrarlo bajo un horizonte E }\end{array}$ \\
\hline $\mathrm{C}$ & $\begin{array}{l}\text { Horizonte mineral que está poco afectado por procesos pedogenéticos; generalmente se ubica en la parte } \\
\text { más profunda del perfil de suelo, sobre la roca }\end{array}$ \\
\hline $\begin{array}{l}\text { Prefijo } \\
2\end{array}$ & $\begin{array}{l}\text { Segunda capa de material (más profunda) que forma un perfil de suelo. Presenta un origen distinto al } \\
\text { de la primera capa }\end{array}$ \\
\hline $\begin{array}{l}\text { Sufijos } \\
1,2,3\end{array}$ & Indican subdivisiones o subhorizontes al interior de un horizonte \\
\hline $\begin{array}{l}\text { Sufijo } \\
\text { g }\end{array}$ & $\begin{array}{l}\text { Símbolo que indica un proceso de reducción del hierro (gleyzación fuerte) por saturación del suelo con } \\
\text { agua; el horizonte muestra una morfología típica (colores) de estos procesos }\end{array}$ \\
\hline $\mathrm{BC}$ & Horizonte de transición entre $\mathrm{B}$ y $\mathrm{C}$, donde dominan las características de $\mathrm{B}$ \\
\hline Profundidad & $\begin{array}{l}\text { El nivel cero desde donde se comienza la medición de la profundidad del suelo corresponde al nivel superior } \\
\text { del primer horizonte mineral, por debajo del mantillo; generalmente corresponde a un horizonte A }\end{array}$ \\
\hline
\end{tabular}

principales características típicas de los horizontes del suelo.

Se realizó un análisis químico del suelo en 14 perfiles $^{6}$. Se tomaron muestras de cada horizonte. En ellas se analizó: pH por determinación electrométrica en suspensión con agua destilada; carbono total $(\%)$ determinado por oxidación mediante dicromato de potasio en medio sulfúrico; nitrógeno total (\%) por digestión con ácido sulfúrico, método Kjeldahl; fósforo disponible ( $\mathrm{mg} \mathrm{kg}^{-}$ $\left.{ }^{1}\right)$ determinado en solución de bicarbonato de sodio a pH 8,5 (método de Olsen), determinación colorimétrica; fracción de elementos extraíbles ( $\left.\mathrm{mg} \mathrm{kg}^{-1}\right)$ sodio, potasio, calcio, magnesio, hierro, manganeso, cobre, zinc y aluminio determinados

\footnotetext{
${ }^{6}$ Métodos según el manual del laboratorio de nutrición y suelos forestales, Instituto de Silvicultura, Universidad Austral de Chile, Valdivia, Chile.
}

en extracto acetato de amonio y DTPA a pH 4,8, por espectrofotometría de absorción atómica; boro $\left(\mathrm{mg} \mathrm{kg}^{-1}\right)$ soluble en agua en ebullición, método azometina $\mathrm{H}$, determinación colorimétrica; aluminio intercambiable $\left(\mathrm{mg} \mathrm{kg}^{-1}\right)$, en extracto de cloruro de potasio $1 \mathrm{M}$, determinado por espectrofotometría de absorción atómica.

Se compararon las medias de grupos de sitios, según distintas características edáficas o topográficas, con la prueba t para dos muestras independientes, considerando sus variaciones de tamaño y varianza (Hartung et al. 2002).

\section{RESULTADOS Y DISCUSIÓN}

Caracterización morfológica y física de los suelos de Río Bueno

Mantillo: El mantillo bajo bosque presentó una variación de 2 a $20 \mathrm{~cm}$ de espesor (promedio $=8,1$ $\mathrm{cm} ; \mathrm{DE}=5,3 \mathrm{~cm} ; \mathrm{n}=28)$; sin embargo, en el 60 
$\%$ de los casos varió entre 4 y $6 \mathrm{~cm}$. No se observaron diferencias del espesor en las distintas ubicaciones en el relieve. El mantillo concentró la mayor proporción de raíces de todo el perfil de suelo, principalmente en los horizonte Oe y Oa, donde generalmente se apreció un fieltro de raicillas (> 50 raicillas $\mathrm{dm}^{-2}$ ). La estructura era suelta en todos los horizontes. Dominan las formas de humus moder a mor.

Suelo mineral: Los perfiles presentaron entre 1 y 4 horizontes. La secuencia de horizontes contempla dos morfologías más comunes: A-B-C y E-2Bhs-C. En el color de los horizontes (E, A, Bhs y B) el tinte (hue) que dominó fue 10 YR y ocasionalmente $2.5 \mathrm{Y}$; en la saturación (chroma) y la intensidad (value) existen mayores variaciones. El horizonte E tenía un color blanco, con una saturación por sobre el valor cinco y una intensidad baja (1 y 2). En los horizontes A y Bhs los colores eran muy parecidos y presentaron una saturación y una intensidad que varió de baja a media (1-4). Los horizontes B mostraron valores de saturación un poco mayores que A y Bhs (2-5). Los colores más oscuros en los horizontes A y Bhs con respecto a los B se explican por la mayor acumulación de materia orgánica en los primeros (Tabla 2 y 5).

En general los suelos eran delgados (Fig. 2); la profundidad de desarrollo varió entre 10 y $105 \mathrm{~cm}$ $(\operatorname{moda}=40 \mathrm{~cm})$. No se observó diferencia $(\mathrm{P}>$

TABLA 2

Características químicas de suelos en perfiles de los sectores Río Bueno y Vicuña

Chemical characteristics of the soils in profiles of Río Bueno y Vicuña

\begin{tabular}{|c|c|c|c|c|c|c|c|c|}
\hline $\begin{array}{l}\text { Perfil, tipo de } \\
\text { bosque y sector }\end{array}$ & Horizonte & $\begin{array}{l}\text { Profundidad } \\
\quad(\mathrm{cm})\end{array}$ & $\begin{array}{c}\mathrm{pH} \\
\text { agua }\end{array}$ & $\begin{array}{c}\mathrm{C} \\
(\%)\end{array}$ & $\begin{array}{c}\mathrm{MO}^{*} \\
(\%)\end{array}$ & $\begin{array}{c}\mathrm{N} \\
(\%)\end{array}$ & $\mathrm{C} / \mathrm{N}$ & $\begin{array}{c}\mathrm{P} \\
\left(\mathrm{mg} \mathrm{kg}^{-1}\right)\end{array}$ \\
\hline 8 & Oi & $11-9$ & 5,09 & - & - & - & - & 7 \\
\hline Nothofagus pumilio & $\mathrm{Oe}$ & $9-0$ & 4,75 & 31,2 & 53,8 & 1,91 & 16 & 1 \\
\hline \multirow{2}{*}{ Río Bueno } & $\mathrm{E}$ & $0-6$ & 4,43 & 1,6 & 2,8 & 0,11 & 15 & 1 \\
\hline & 2Bhs & $6-22$ & 4,84 & 11,3 & 19,5 & 0,66 & 17 & 1 \\
\hline 9 & Oi & $7-6$ & 4,66 & - & - - & - & 29 & \\
\hline Nothofagus betuloides & $\mathrm{Oe} / \mathrm{Oa}$ & $6-0$ & 5,06 & - & $-1,12$ & - & 52 & \\
\hline Nothofagus pumilio & E & $0-11$ & 4,59 & 1,2 & 2,1 & 0,11 & 11 & 2 \\
\hline Río Bueno & 2Bhs & $11-20$ & 4,93 & 4,5 & 7,8 & 0,34 & 13 & 1 \\
\hline 1 & Oe & $7-5$ & 5,08 & - & - & - & 46 & \\
\hline Nothofagus betuloides & $\mathrm{Oa}$ & $5-0$ & 4,97 & 21,0 & 36,2 & 1,39 & 15 & 9 \\
\hline Nothofagus pumilio & $\mathrm{A}$ & $0-15$ & 5,06 & 4,4 & 7,6 & 0,41 & 11 & 41 \\
\hline \multirow[t]{2}{*}{ Río Bueno } & $\mathrm{Bg} 1$ & $15-71$ & 5,42 & 0,7 & 1,2 & 0,09 & 8 & 5 \\
\hline & $\mathrm{Bg} 2$ & $71-105$ & - & 0,8 & 1,4 & 0,10 & 8 & 5 \\
\hline 2 & Oi & $15-13$ & 5,47 & - & - & - & 45 & \\
\hline Nothofagus betuloides & $\mathrm{Oe}$ & $13-6$ & 5,44 & 28,0 & 48,3 & 1,38 & 20 & 35 \\
\hline Drimis winterii & $\mathrm{Oa}$ & $6-0$ & 5,09 & - & $-0,44$ & - & 5 & \\
\hline Maitenus magallanica & $\mathrm{A}$ & $0-12$ & 4,48 & 3,5 & 6,0 & 0,09 & 39 & 2 \\
\hline \multirow[t]{3}{*}{ Río Bueno } & B1 & $12-23$ & 4,53 & 4,0 & 6,9 & 0,25 & 16 & $<1$ \\
\hline & $\mathrm{B} 2$ & $23-31$ & 4,09 & 3,7 & 6,4 & 0,17 & 22 & 14 \\
\hline & $\mathrm{C}$ & $31-35$ & 5,05 & 0,8 & 1,4 & 0,47 & 2 & 3 \\
\hline 12 & $\mathrm{Oa}$ & $4-0$ & 5,64 & - & $-0,94$ & - & 33 & \\
\hline Nothofagus pumilio & $\mathrm{E}$ & $0-5$ & 5,36 & 4,1 & 7,1 & 0,35 & 12 & 40 \\
\hline \multirow[t]{3}{*}{ Vicuña } & $2 \mathrm{Bhs}$ & $5-14$ & 5,02 & 4,7 & 8,1 & 0,53 & 9 & 4 \\
\hline & B & $14-20$ & 5,56 & 4,5 & 7,8 & 0,39 & 12 & 1 \\
\hline & $\mathrm{BC}$ & $20-29$ & 4,60 & 4,8 & 8,3 & 0,30 & 16 & 1 \\
\hline 13 & $\mathrm{E}$ & $0-9$ & 4,74 & 0,7 & 1,2 & 0,01 & 70 & 1 \\
\hline Nothofagus pumilio & 2Bhs & $9-17$ & 4,77 & 7,7 & 13,3 & 0,33 & 23 & $<1$ \\
\hline Vicuña & $\mathrm{BC}$ & $17-27$ & 5,19 & 9,2 & 15,9 & 0,44 & 21 & $<1$ \\
\hline 14 & $\mathrm{Oe} / \mathrm{Oa}$ & $5-0$ & 5,02 & 20,8 & 35,9 & 0,90 & 23 & 36 \\
\hline Nothofagus pumilio & E & $0-5$ & 4,66 & 2,1 & 3,6 & 0,12 & 18 & 3 \\
\hline \multirow[t]{2}{*}{ Vicuña } & $2 \mathrm{Bhs}$ & $5-15$ & 4,69 & 11,8 & 20,3 & 0,07 & 169 & $<1$ \\
\hline & $\mathrm{BC}$ & $15-34$ & 4,95 & 13,05 & 23,3 & 0,46 & 29 & $<1$ \\
\hline
\end{tabular}

*Materia orgánica; (-) no determinado 


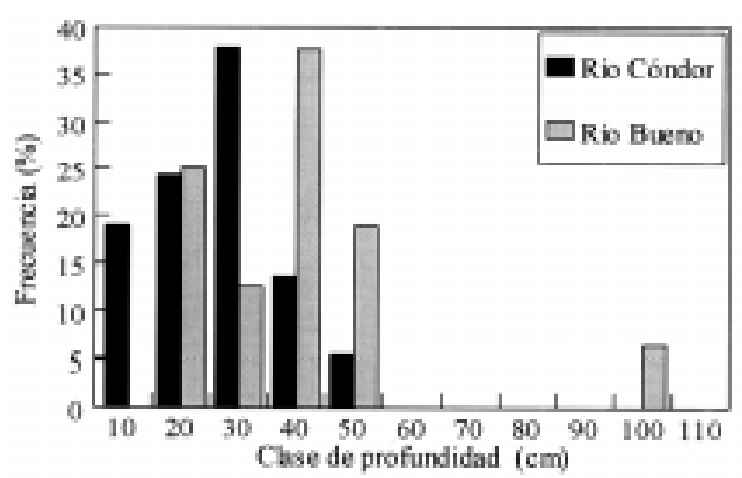

Fig. 2: Frecuencia de la profundidad de desarrollo del suelo en Río Bueno y Río Cóndor.

Frequency of the development depth of the soil in Río Bueno and Río Cóndor.

$0,05)$ de profundidad entre las distintas ubicaciones en el relieve. La pedregosidad era moderada a alta (15-50\%), siendo evidente el aumento a mayor profundidad. El arraigamiento es menor que en el mantillo, disminuyendo con la profundidad. El horizonte E tenía un escaso arraigamiento, pero ocasionalmente abundante a muy abundante, asociado a una mayor proporción de $N$. pumilio en el bosque y a suelos de mejor drenaje. La estructura de los horizontes era generalmente masiva a granular; el horizonte E presentó generalmente una estructura masiva. La consistencia era friable en todos los horizontes. Los horizontes superiores (E, A y Bhs) tenían textura franco limosa, mientras que los más profundos eran de textura franco arenosa o franco arcillosa. La capacidad de agua aprovechable era generalmente baja, no superando los $60 \mathrm{~mm}$ en el perfil de suelo. El horizonte E presentó menor capacidad de agua aprovechable. El drenaje interno era moderado en las laderas, mientras que en los planos era generalmente lento.

Caracterización morfológica y física de los suelos de Río Cóndor

Mantillo: los suelos de Río Cóndor presentaron mantillo con características similares a las encontradas en Río Bueno. Su espesor varió entre 1,5 y $30 \mathrm{~cm}$ (promedio $=7,7 \mathrm{~cm} ; \mathrm{DE}=5,4 \mathrm{~cm} ; \mathrm{n}=56$ ), pero en el $60 \%$ de los casos se encontró entre 4 y $10 \mathrm{~cm}$. Las formas de humus dominantes son moder y mor.

Suelo mineral: El número de horizontes varió entre 1 y 5 . Las secuencias más típicas fueron las mismas observadas en Río Bueno. Estas secuencias se presentaron en todas las ubicaciones del relieve, incluso en terrazas marinas. Sin embar- go, las terrazas de río no mostraron la secuencia antes descrita, sino más bien presentaron capas geológicas con escasa evolución. Estas capas diferían de manera importante en cuanto a profundidad y pedregosidad. Los colores fueron similares a los descritos para el sector Río Bueno.

En general, son suelos más delgados que en Río Bueno, con una profundidad entre 5 y $48 \mathrm{~cm}$ $($ moda $=30 \mathrm{~cm})($ Fig. 2$)$. Los volúmenes de pedregosidad, arraigamiento y color fueron similares a los descritos para Río Bueno. La estructura fue muy variada, desde masiva hasta formas redondeadas y cúbicas; lo más frecuente es la masiva a granular. La estructura del horizonte $\mathrm{E}$ es masiva. La consistencia aumentó en profundidad, desde muy friable hasta firme en los horizontes inferiores. La textura fue franca limosa en los horizontes superficiales (E, A, Bhs y a veces B), y franca arenosa en los horizontes B y C. La capacidad de agua aprovechable presentó el mismo comportamiento que en Río Bueno. El drenaje interno del suelo varió de moderado a lento, siendo el drenaje moderado el más frecuente.

\section{Tipos de suelos}

En muchos perfiles se pudo constatar la presencia de un horizonte de color blanco (horizonte E), ubicado inmediatamente debajo del mantillo, de textura franco limosa, estructura masiva y escasa pedregosidad y arraigamiento. Esta última característica puede explicarse a través de su análisis químico (Tabla 2-7), ya que presentó una alta acidez, alta concentración de aluminio (saturación) y un nivel medio a bajo de nutrientes, comparable con el horizonte C. Por otra parte, de su estructura masiva y cerrada se infiere que este horizonte no es favorable para el buen desarrollo de raíces.

Díaz et al. (1960), Pisano (1977) y Sadzawka et al.(1995) han descrito este horizonte blanco como un horizonte eluvial, producto de procesos de podzolización que estarían ocurriendo en la zona. Otras publicaciones (INTA-ORSTON 1991, Thiers 1997) no son explícitos en la clasificación taxonómica del horizonte, por lo cual plantean la hipótesis que su origen es volcánico (depósito de cenizas), combinado con procesos de podzolización (eluviación). Con los datos analizados en este trabajo y otros (Sadzawka et al. 1995, Arroyo et al. 19967, F. Colmet-Daage, recopilación inédita de datos y W. Luzio comunicación personal) esta última hipótesis sería razonable.

\footnotetext{
${ }^{7}$ Op. cit.
} 
De la comparación de los perfiles que presentaron este horizonte blanco, identificado como horizonte $\mathrm{E}$, con aquellos que no lo presentaron en el área de estudio (Tabla 2-7, Fig. 3), se infiere que existen procesos de podzolización. En la Fig.3 se observa que la proporción B/A, definida por el cuociente entre las concentraciones del horizonte subsuperficial (tipo B) y del superior (tipo A o E), es significativamente mayor para los elementos carbono, nitrógeno, sodio, aluminio, hierro y cobre en los perfiles de suelo con horizonte $\mathrm{E}$ en comparación con los perfiles sin dicho horizonte. Esta proporción $\mathrm{B} / \mathrm{A}$, en los niveles alcanzados para los elementos mencionados, es un indicador de procesos de eluviación-iluviación característicos de los suelos Spodosoles (United States Departament of Agriculture 1999).

En los horizontes E se evidenciaría, entonces, la eluviación a través de los menores niveles de carbono, nitrógeno y elementos minerales, especialmente sodio, cobre, hierro y aluminio. Consecuentemente, se aprecia la acumulación correspondiente en los horizontes inferiores (Bhs), siendo más clara para los elementos hierro y aluminio (Fig.3). Sin embargo, las texturas presentaron un contenido relativamente alto de arcillas en el

TABLA 3

Elementos minerales disponibles de suelos en perfiles de los sectores Río Bueno y Vicuña

Available fraction of the soils in profiles of Río Bueno y Vicuña

\begin{tabular}{|c|c|c|c|c|c|c|c|c|c|c|c|c|}
\hline $\begin{array}{l}\text { Perfil, tipo de } \\
\text { bosque y sector }\end{array}$ & $\begin{array}{l}\text { Hori- F } \\
\text { zonte }\end{array}$ & $\begin{array}{l}\text { rofundidad } \\
\qquad(\mathrm{cm})\end{array}$ & $\mathrm{Na}$ & K & $\mathrm{Ca}$ & $\mathrm{Mg}$ & $\mathrm{Al}$ & $\begin{array}{r}\mathrm{Fe} \\
\left.\mathrm{g} \mathrm{kg}^{-1}\right)\end{array}$ & $\mathrm{Mn}$ & $\mathrm{Cu}$ & $\mathrm{Zn}$ & B \\
\hline 8 & Oi & $11-9$ & 196 & 990 & 10.280 & 1562 & 16 & 92 & 940 & 10,4 & 138,0 & - \\
\hline Nothofagus pumilio & Oe & $9-0$ & 166 & 206 & 6.160 & 804 & 12 & 136 & 330 & 5,8 & 62,0 & - \\
\hline \multirow[t]{2}{*}{ Río Bueno } & $\mathrm{E}$ & $0-6$ & 60 & 94 & 894 & 280 & 390 & 70 & 14 & 3,0 & 5,2 & 0,9 \\
\hline & 2Bhs & $6-22$ & 106 & 32 & 448 & 172 & 3.520 & 1.326 & 2 & 7,2 & 1,2 & 0,3 \\
\hline 9 & Oi & $7-6$ & 182 & 1.228 & 8.640 & 1.400 & 14 & 46 & 1.610 & 15,0 & 78,0 & - \\
\hline Nothofagus betuloides & $\mathrm{Oe} / \mathrm{Oa}$ & $6-0$ & 108 & 548 & 7.480 & 1.204 & 48 & 408 & 2.824 & 8,4 & 84,0 & - \\
\hline Nothofagus pumilio & $\mathrm{E}$ & $0-11$ & 38 & 136 & 390 & 112 & 504 & 100 & 24 & 2,8 & 5,2 & 0,3 \\
\hline Río Bueno & 2Bhs & $11-20$ & 40 & 36 & 58 & 24 & 3.986 & 728 & 6 & 5,4 & 0,6 & 0,3 \\
\hline 1 & Oe & $7-5$ & 172 & 1.358 & 7.960 & 1.654 & 170 & 266 & 844 & 12,2 & 88,0 & - \\
\hline Nothofagus betuloides & $\mathrm{Oa}$ & $5-0$ & 256 & 362 & 1.810 & 698 & 2.204 & 546 & 356 & 13,8 & 16,0 & 1,4 \\
\hline Nothofagus pumilio & $\mathrm{A}$ & $0-15$ & 128 & 106 & 684 & 334 & 2.572 & 614 & 6 & 11,6 & 6,2 & 0,2 \\
\hline \multirow[t]{2}{*}{ Río Bueno } & $\mathrm{Bg} 1$ & $15-71$ & 100 & 86 & 1.158 & 646 & 420 & 178 & 68 & 7,6 & 1,0 & $<0,1$ \\
\hline & $\mathrm{Bg} 2$ & $71-105$ & 154 & 146 & 2.278 & 666 & 118 & 254 & 8 & 15,6 & 3,0 & $<0,1$ \\
\hline 2 & Oi & $15-13$ & 536 & 1.776 & 7.656 & 1.704 & 70 & 406 & 1.736 & 12,4 & 36,0 & - \\
\hline Nothofagus betuloides & Oe & $13-6$ & 472 & 618 & 10.120 & 1.914 & 62 & 614 & 1.420 & 7,4 & 32,0 & 7,9 \\
\hline Drimis winterii & $\mathrm{Oa}$ & $6-0$ & 376 & 364 & 3.668 & 818 & 576 & 852 & 1.092 & 5,6 & 10,2 & 3,2 \\
\hline Maitenus magallanica & $\mathrm{A}$ & $0-12$ & 286 & 76 & 970 & 308 & 1.896 & 774 & 6 & 5,2 & 0,2 & 1,5 \\
\hline \multirow[t]{3}{*}{ Río Bueno } & B1 & $12-23$ & 294 & 26 & 536 & 204 & 3.066 & 762 & 2 & 4,6 & 0,2 & 1,1 \\
\hline & B2 & $23-31$ & 244 & 20 & 244 & 162 & 2.494 & 774 & 2 & 3,0 & 2,6 & 0,8 \\
\hline & $\mathrm{C}$ & $31-35$ & 126 & 16 & 148 & 92 & 1.076 & 336 & 2 & 3,0 & 0,2 & 0,1 \\
\hline 12 & $\mathrm{Oa}$ & $4-0$ & 52 & 606 & 6.376 & 892 & 84 & 454 & 778 & 4,6 & 100,0 & - \\
\hline Nothofagus pumilio & $\mathrm{E}$ & $0-5$ & 44 & 424 & 1.994 & 380 & 596 & 556 & 352 & 3,0 & 16,0 & - \\
\hline \multirow[t]{3}{*}{ Vicuña } & 2Bhs & $5-14$ & 32 & 136 & 284 & 50 & 6.458 & 2.764 & 32 & 8,0 & 0,4 & 0,2 \\
\hline & B & $14-20$ & 38 & 24 & 36 & 16 & 5.668 & 542 & 6 & 6,4 & 0,2 & $<0,1$ \\
\hline & $\mathrm{BC}$ & $20-29$ & 42 & 16 & 32 & 18 & 4.438 & 360 & 6 & 4,2 & 0,2 & $<0,1$ \\
\hline 13 & E & $0-9$ & 54 & 32 & 52 & 10 & 1.192 & 326 & 4 & 3,6 & 0,2 & - \\
\hline Nothofagus pumilio & 2Bhs & $9-17$ & 68 & 28 & 48 & 10 & 5.674 & 1.218 & 8 & 5,4 & 0,2 & - \\
\hline Vicuña & $\mathrm{BC}$ & $17-27$ & 40 & 28 & 40 & 12 & 5.170 & 538 & 4 & 3,4 & 0,2 & - \\
\hline 14 & $\mathrm{Oe} / \mathrm{Oa}$ & $5-0$ & 70 & 368 & 488 & 590 & 20 & 292 & 3.126 & 7,0 & 66,0 & - \\
\hline Nothofagus pumilio & $\mathrm{E}$ & $0-5$ & 24 & 154 & 588 & 116 & 194 & 168 & 680 & 3,6 & 7,6 & 0,2 \\
\hline \multirow{2}{*}{ Vicuña } & 2Bhs & $5-15$ & 30 & 102 & 126 & 64 & 3.404 & 1.092 & 10 & 3,8 & 2,4 & 0,2 \\
\hline & $\mathrm{BC}$ & $15-34$ & 24 & 32 & 58 & 16 & 4.356 & 444 & 2 & 5,2 & 3,2 & $<0,1$ \\
\hline
\end{tabular}

(-) No determinado 
TABLA 4

Bases y aluminio de suelos en perfiles de los sectores Río Bueno y Vicuña

Bases and aluminum in the soils in profiles of Río Bueno y Vicuña

\begin{tabular}{|c|c|c|c|c|c|c|c|c|c|}
\hline $\begin{array}{l}\text { Perfil, tipo } \\
\text { de bosque } \\
\text { y sector }\end{array}$ & Horizonte & $\begin{array}{l}\text { Profundidad } \\
\qquad(\mathrm{cm})\end{array}$ & $\mathrm{Na}$ & $\mathrm{K}$ & $\begin{array}{l}\mathrm{Ca} \\
{[\mathrm{cmol}} \\
\end{array}$ & $\mathrm{Mg}$ & $\begin{array}{c}\text { Suma } \\
\text { de bases }\end{array}$ & $\begin{array}{l}\mathrm{Al} \\
\text { Intercam- } \\
\text { biable }\end{array}$ & $\begin{array}{l}\text { Saturación } \\
\text { de } \mathrm{Al} \\
(\%)\end{array}$ \\
\hline $\begin{array}{l}8 \\
\text { Nothofagus pumilio } \\
\text { Río Bueno }\end{array}$ & $\begin{array}{c}\mathrm{Oi} \\
\mathrm{Oe} \\
\mathrm{E} \\
2 \mathrm{Bhs}\end{array}$ & $\begin{array}{l}11-9 \\
9-0 \\
0-6 \\
6-22\end{array}$ & $\begin{array}{l}0,85 \\
0,72 \\
0,26 \\
0,46\end{array}$ & $\begin{array}{l}2,53 \\
0,53 \\
0,24 \\
0,08\end{array}$ & $\begin{array}{r}51,40 \\
30,80 \\
4,47 \\
2,24\end{array}$ & $\begin{array}{r}12,91 \\
6,64 \\
2,31 \\
1,42\end{array}$ & $\begin{array}{r}67,69 \\
38,69 \\
7,29 \\
4,20\end{array}$ & $\begin{array}{r}- \\
0,67 \\
5,89 \\
10,22\end{array}$ & $\begin{array}{r}- \\
1,7 \\
44,7 \\
70,9\end{array}$ \\
\hline $\begin{array}{l}9 \\
\text { Nothofagus betuloides } \\
\text { Nothofagus pumilio } \\
\text { Río Bueno }\end{array}$ & $\begin{array}{c}\mathrm{Oi} \\
\mathrm{Oe} / \mathrm{Oa} \\
\mathrm{E} \\
2 \mathrm{Bhs}\end{array}$ & $\begin{array}{c}7-6 \\
6-0 \\
0-11 \\
11-20\end{array}$ & $\begin{array}{l}0,79 \\
0,47 \\
0,17 \\
0,17\end{array}$ & $\begin{array}{l}3,14 \\
1,40 \\
0,35 \\
0,09\end{array}$ & $\begin{array}{r}43,20 \\
37,40 \\
1,95 \\
0,29\end{array}$ & $\begin{array}{r}11,57 \\
9,95 \\
0,93 \\
0,20\end{array}$ & $\begin{array}{r}58,70 \\
49,22 \\
3,39 \\
0,75\end{array}$ & $\begin{array}{l}1,56 \\
0,69 \\
6,18 \\
7,29\end{array}$ & $\begin{array}{r}2,6 \\
1,4 \\
64,6 \\
90,6\end{array}$ \\
\hline $\begin{array}{l}1 \\
\text { Nothofagus betuloides } \\
\text { Nothofagus pumilio } \\
\text { Río Bueno }\end{array}$ & $\begin{array}{c}\mathrm{Oe} \\
\mathrm{Oa} \\
\mathrm{A} \\
\mathrm{Bg} 1 \\
\mathrm{Bg} 2\end{array}$ & $\begin{array}{c}7-5 \\
5-0 \\
0-15 \\
15-71 \\
71-105\end{array}$ & $\begin{array}{l}0,75 \\
1,11 \\
0,56 \\
0,43 \\
0,67\end{array}$ & $\begin{array}{l}3,47 \\
0,93 \\
0,27 \\
0,22 \\
0,37\end{array}$ & $\begin{array}{r}39,80 \\
9,05 \\
3,42 \\
5,79 \\
11,39\end{array}$ & $\begin{array}{r}13,67 \\
5,77 \\
2,76 \\
5,34 \\
5,50\end{array}$ & $\begin{array}{r}57,69 \\
16,86 \\
7,01 \\
11,78 \\
17,94\end{array}$ & $\begin{array}{r}- \\
2,29 \\
11,07 \\
3,93 \\
0,87\end{array}$ & $\begin{array}{r}- \\
12,0 \\
61,2 \\
25,0 \\
4,6\end{array}$ \\
\hline $\begin{array}{l}2 \\
\text { Nothofagus betuloides } \\
\text { Drimis winterii } \\
\text { Maitenus magallanica } \\
\text { Río Bueno }\end{array}$ & $\begin{array}{c}\text { Oi } \\
\text { Oe } \\
\text { Oa } \\
\text { A } \\
\text { B1 } \\
\text { B2 } \\
\text { C }\end{array}$ & $\begin{array}{c}15-13 \\
13-6 \\
6-0 \\
0-12 \\
12-23 \\
23-31 \\
31-35\end{array}$ & $\begin{array}{l}2,33 \\
2,05 \\
1,63 \\
1,24 \\
1,28 \\
1,06 \\
0,55\end{array}$ & $\begin{array}{l}4,54 \\
1,58 \\
0,93 \\
0,19 \\
0,07 \\
0,05 \\
0,04\end{array}$ & $\begin{array}{r}38,28 \\
50,60 \\
18,34 \\
4,85 \\
2,68 \\
1,22 \\
0,74\end{array}$ & $\begin{array}{r}14,08 \\
15,82 \\
6,76 \\
2,55 \\
1,69 \\
1,34 \\
0,76\end{array}$ & $\begin{array}{r}59,24 \\
70,05 \\
27,67 \\
8,83 \\
5,71 \\
3,67 \\
2,09\end{array}$ & $\begin{array}{l}0,80 \\
0,73 \\
0,87 \\
6,27 \\
8,87 \\
7,91 \\
4,60\end{array}$ & $\begin{array}{r}1,3 \\
1,0 \\
3,0 \\
41,5 \\
60,8 \\
68,3 \\
68,8\end{array}$ \\
\hline $\begin{array}{l}12 \\
\text { Nothofagus pumilio } \\
\text { Vicuña }\end{array}$ & $\begin{array}{l}\text { Oa } \\
\text { E } \\
2 \mathrm{Bhs} \\
\mathrm{B} \\
\mathrm{BC}\end{array}$ & $\begin{array}{c}4-0 \\
0-5 \\
5-14 \\
14-20 \\
20-29\end{array}$ & $\begin{array}{l}0,23 \\
0,19 \\
0,14 \\
0,17 \\
0,18\end{array}$ & $\begin{array}{l}1,55 \\
1,08 \\
0,35 \\
0,06 \\
0,04\end{array}$ & $\begin{array}{r}31,88 \\
9,97 \\
1,42 \\
0,18 \\
0,16\end{array}$ & $\begin{array}{l}7,37 \\
3,14 \\
0,41 \\
0,13 \\
0,15\end{array}$ & $\begin{array}{r}41,03 \\
14,39 \\
2,32 \\
0,54 \\
0,53\end{array}$ & $\begin{array}{l}- \\
2,62 \\
3,73 \\
4,02 \\
4,18\end{array}$ & $\begin{array}{l}- \\
15,4 \\
61,7 \\
88,2 \\
88,7\end{array}$ \\
\hline $\begin{array}{l}13 \\
\text { Nothofagus pumilio } \\
\text { Vicuña }\end{array}$ & $\begin{array}{c}\mathrm{E} \\
2 \mathrm{Bhs} \\
\mathrm{BC}\end{array}$ & $\begin{array}{c}0-9 \\
9-17 \\
17-27\end{array}$ & $\begin{array}{l}0,23 \\
0,30 \\
0,17\end{array}$ & $\begin{array}{l}0,08 \\
0,07 \\
0,07\end{array}$ & $\begin{array}{l}0,26 \\
0,24 \\
0,20\end{array}$ & $\begin{array}{l}0,08 \\
0,08 \\
0,10\end{array}$ & $\begin{array}{l}0,66 \\
0,69 \\
0,54\end{array}$ & $\begin{array}{r}- \\
1,27 \\
0,78\end{array}$ & $\begin{array}{r}- \\
64,7 \\
58,8\end{array}$ \\
\hline $\begin{array}{l}14 \\
\text { Nothofagus pumilio } \\
\text { Vicuña }\end{array}$ & $\begin{array}{l}\mathrm{Oe} / \mathrm{Oa} \\
\mathrm{E} \\
2 \mathrm{Bhs} \\
\mathrm{BC}\end{array}$ & $\begin{array}{c}5-0 \\
0-5 \\
5-15 \\
15-34\end{array}$ & $\begin{array}{l}0,30 \\
0,10 \\
0,13 \\
0,10\end{array}$ & $\begin{array}{l}0,94 \\
0,39 \\
0,26 \\
0,08\end{array}$ & $\begin{array}{l}2,44 \\
2,94 \\
0,63 \\
0,29\end{array}$ & $\begin{array}{l}4,88 \\
0,96 \\
0,53 \\
0,13\end{array}$ & $\begin{array}{l}8,56 \\
4,40 \\
1,55 \\
0,61\end{array}$ & $\begin{array}{l}0,69 \\
2,64 \\
9,04 \\
4,09\end{array}$ & $\begin{array}{r}7,4 \\
37,6 \\
85,4 \\
87,0\end{array}$ \\
\hline
\end{tabular}

(-) No determinado

horizonte blanco, lo cual no es típico de un horizonte eluviado y sugiere depósitos volcánicos. Es necesario determinar analíticamente la textura para verificar la participación de arcillas.

No obstante que en la zona existan procesos de podzolización, estos no serían la causa exclusiva del color blanco del horizonte E. Se plantea la hipótesis de que se trataría de un material con distinto origen, debido a que dicho horizonte presenta una textura y estructura uniforme, carencia casi total de pedregosidad, excepto por fenómenos coluviales y de erosión, una alta frecuencia en terrenos de menor elevación y una orientación preferentemente norte. Además, es más frecuente su presencia bajo bosque, que en la estepa. Estas características refuerzan la hipótesis de un origen volcánico de esta capa de suelo superficial.

Esta región tuvo una fuerte influencia volcánica, identificándose por lo menos cuatro volcanes de actividad reciente (González-Ferrán 1995). Se pueden reconocer varias capas de ceniza volcánica depositadas en Tierra de Fuego, cuyo origen está ligado a la actividad de los volcanes Burney o Reclus, localizados al norte de la Patagonia, o de otro volcán localizado en la isla Gordon (INTAORSTON 1991, Arroyo et al. $\left.1996^{8}\right)$. Si a este

${ }^{8}$ Op. cit. 




Fig. 3: Proporción de concentraciones de elementos entre el horizonte inferior (tipo B) y el horizonte superior $($ tipo A o E); $(\mathrm{Al}-\mathrm{i})=$ aluminio intercambiable $;(*)=$ diferencia significativa $(\mathrm{P}<0,05)$ entre ambos grupos de perfiles.

Proportion of concentrations of the elements between the inferior horizon (type B) and upper horizon (type A or E); (Al-I) $=$ aluminum of interchange $; *)=$ significant difference $(\mathrm{P}<0.05)$ between both groups of profiles.

TABLA 5

Características químicas de suelos en perfiles del sector Río Cóndor

Chemical characteristics of soils in profiles of Río Cóndor

\begin{tabular}{|c|c|c|c|c|c|c|c|c|}
\hline $\begin{array}{l}\text { Perfil, tipo de } \\
\text { bosque,y sector }\end{array}$ & Horizonte & $\begin{array}{l}\text { Profundidad } \\
\qquad(\mathrm{cm})\end{array}$ & $\begin{array}{l}\mathrm{pH} \\
\text { agua }\end{array}$ & $\begin{array}{c}\mathrm{C} \\
(\%)\end{array}$ & $\begin{array}{l}\text { MO* } \\
(\%)\end{array}$ & $\begin{array}{l}\mathrm{N} \\
(\%)\end{array}$ & $\mathrm{C} / \mathrm{N}$ & $\begin{array}{c}\mathrm{P} \\
\left(\mathrm{mg} \mathrm{kg}^{-1}\right)\end{array}$ \\
\hline 3 & $\mathrm{Oa}$ & 12 & 6,20 & 20,0 & 34,5 & 1,17 & 17 & 52 \\
\hline Nothofagus betuloides & A & $0-25$ & 4,85 & 7,0 & 12,1 & 0,33 & 21 & 8 \\
\hline \multirow[t]{2}{*}{ Nothofagus pumilio } & Bhs & $25-35$ & 4,53 & 10,1 & 17,4 & 0,46 & 22 & 7 \\
\hline & $\mathrm{C}$ & $35-45$ & 5,06 & 1,5 & 2,6 & 0,07 & 21 & 6 \\
\hline 4 & $\mathrm{ACg}$ & $0-8$ & 4,82 & 5,5 & 9,5 & 0,05 & 110 & 11 \\
\hline Nothofagus betuloides & $\mathrm{C}$ & $8-20$ & 4,41 & 3,0 & 5,2 & 0,14 & 21 & 6 \\
\hline 5 & A & $0-6$ & 3,25 & 4,6 & 7,9 & 0,33 & 14 & 13 \\
\hline Nothofagus betuloides & B & $6-30$ & 3,39 & 33,0 & 56,9 & 0,75 & 44 & 11 \\
\hline Nothofagus pumilio & $\mathrm{C}$ & $30-32$ & 4,11 & 2,4 & 4,1 & 0,14 & 17 & 23 \\
\hline 6 & A & $0-20$ & 4,49 & 4,3 & 7,4 & 0,18 & 24 & 1 \\
\hline Nothofagus betuloides & $\mathrm{C}$ & $20-40$ & 5,58 & 1,6 & 2,8 & 0,14 & 11 & 1 \\
\hline 7 & Oi & $7-5$ & 5,18 & - & - & - & - & 34 \\
\hline \multirow[t]{5}{*}{ Nothofagus pumilio } & $\mathrm{Oe} / \mathrm{Oa}$ & $5-0$ & 6,33 & 10,0 & 17,2 & 0,24 & 42 & 70 \\
\hline & A & $0-6$ & 4,55 & 2,8 & 4,8 & 0,23 & 12 & 3 \\
\hline & B1 & $6-30$ & 4,79 & 2,1 & 3,6 & 0,23 & 9 & 4 \\
\hline & B2 & $30-60$ & 5,02 & 0,6 & 1,0 & 0,53 & 1 & $<1$ \\
\hline & B3 & $60-80$ & 5,28 & 2,4 & 4,1 & 0,01 & 240 & $<1$ \\
\hline 10 & $\mathrm{Oi} / \mathrm{Oe}$ & $10-4$ & 5,59 & 21,4 & 36,9 & - & - & 76 \\
\hline \multirow[t]{3}{*}{ Nothofagus pumilio } & $\mathrm{Oa}$ & $4-0$ & 5,12 & - & - & 0,90 & - & 16 \\
\hline & E & $0-7$ & 4,92 & 1,8 & 3,1 & 0,12 & 15 & 1 \\
\hline & $2 \mathrm{BC}$ & $7-16$ & 5,46 & 1,6 & 2,8 & 0,07 & 23 & 1 \\
\hline 11 & A & $0-5$ & 4,87 & 7,4 & 12,8 & 0,83 & 9 & 2 \\
\hline \multirow[t]{3}{*}{ Pradera } & E & $5-10$ & 5,07 & 2,8 & 4,8 & 0,55 & 5 & 4 \\
\hline & 2Bhs & $10-30$ & 4,98 & 5,0 & 8,6 & 0,22 & 23 & $<1$ \\
\hline & $\mathrm{BC}$ & $30-35$ & 5,11 & 2,3 & 4,0 & 0,23 & 10 & 1 \\
\hline
\end{tabular}

(*) Materia orgánica; (-) no determinado 
fenómeno de vulcanismo se agregan dos factores que son muy importantes en esta zona, como lo es el viento y la geomorfología, se obtienen condiciones adecuadas para que se produzca no sólo el depósito de cenizas volcánicas, sino que además la sedimentación de material fino proveniente de otros sitios.

Al respecto, F. Colmet-Daage (recopilación inédita de datos) plantea para la Patagonia un modelo de formación de suelo, en que el viento y la topografía podrían explicar la ubicación (orientación y frecuencia) del horizonte blanco y respaldar la hipótesis de que su característica de color no sólo es producto de los procesos de podzolización ya comprobados. El modelo plantea que las cenizas o material fino han sido acumulados sobre las vertientes de las lomas abrigadas de los vientos dominantes, que en este caso son las vertientes con exposición preferentemente norte, y sobre la parte baja de las vertientes expuestas al viento (Fig. 4).

De acuerdo a lo anterior, la secuencia más frecuente de horizontes es la siguiente: con horizonte blanco, O-E-2Bhs-C; sin horizonte blanco, OA-B-C y O-A-Bhs-C. Por lo tanto, en la zona se presentan dos órdenes de suelos (Soil Conservation Service 1982): Spodosol e Inceptisol. Los Spodosoles en la zona de estudio comprenden dos sub-ordenes: Aquods, corresponde a suelos que se ubican en terrenos de baja elevación y pendiente suave, donde existen frecuentemente problemas de drenaje, y Cryods que comprende el resto de los suelos de este orden que se desarrollan en terrenos que no presentan problemas de drenaje y bajo la principal influencia de un régimen de temperatura frío. Los suelos restantes pertenecen al orden Inceptisol, se ubican dentro del gran grupo de los Cryochrepts, los que se caracterizan

TABLA 6

Elementos minerales disponibles de suelos en perfiles del sector Río Cóndor

Available fraction of the soils in profiles of Río Cóndor

\begin{tabular}{|c|c|c|c|c|c|c|c|c|c|c|c|c|}
\hline $\begin{array}{l}\text { Perfil, tipo de } \\
\text { bosque y sector }\end{array}$ & $\begin{array}{l}\text { Hori- } \\
\text { zonte }\end{array}$ & $\begin{array}{l}\text { Profundidad } \\
\qquad(\mathrm{cm})\end{array}$ & $\mathrm{Na}$ & $\mathrm{K}$ & $\mathrm{Ca}$ & $\mathrm{Mg}$ & & $\begin{array}{r}\mathrm{Fe} \\
\left.\mathrm{g} \mathrm{kg}^{-1}\right)\end{array}$ & $\mathrm{Mn}$ & $\mathrm{Cu}$ & $\mathrm{Zn}$ & B \\
\hline 3 & $\mathrm{Oa}$ & 12 & 330 & 772 & 10.580 & 1.286 & 360 & 1.260 & 1.284 & 8,4 & 66,0 & - \\
\hline Nothofagus betuloides & A & $0-25$ & 138 & 206 & 920 & 104 & 4.096 & 650 & 52 & 9,6 & 4,8 & 0,2 \\
\hline \multirow[t]{2}{*}{ Nothofagus pumilio } & Bhs & $25-35$ & 56 & 74 & 260 & 72 & 4.438 & 1.380 & 18 & 8,0 & 6,0 & 0,2 \\
\hline & $\mathrm{C}$ & $35-45$ & 14 & 44 & 60 & 42 & 722 & 238 & 12 & 2,2 & 1,8 & $<0,1$ \\
\hline 4 & Acg & $0-8$ & 42 & 68 & 320 & 126 & 692 & 438 & 14 & 4,2 & 2,8 & 1,5 \\
\hline Nothofagus betuloides & $\mathrm{C}$ & $8-20$ & 46 & 66 & 1.020 & 360 & 1.040 & 1.160 & 34 & 3,6 & 2,2 & - \\
\hline 5 & A & $0-6$ & 136 & 78 & 200 & 152 & 892 & 392 & 4 & 4,0 & 5,2 & 1,6 \\
\hline Nothofagus betuloides & B & $6-30$ & 120 & 34 & 114 & 66 & 2.978 & 510 & 2 & 5,0 & 2,6 & 0,7 \\
\hline Nothofagus pumilio & $\mathrm{C}$ & $30-32$ & 50 & 34 & 148 & 70 & 1.748 & 724 & 2 & 14,0 & 5,2 & - \\
\hline 6 & A & $0-20$ & 110 & 60 & 218 & 92 & 2.642 & 516 & 4 & 7,0 & 7,2 & 0,4 \\
\hline Nothofagus betuloides & $\mathrm{C}$ & $20-40$ & 72 & 50 & 956 & 178 & 648 & 416 & 12 & 4,6 & 2,6 & $<0,1$ \\
\hline 7 & Oi & $7-5$ & 174 & 650 & 10.880 & 1.560 & 114 & 376 & 992 & 12,4 & 86,0 & - \\
\hline \multirow[t]{5}{*}{ Nothofagus pumilio } & $\mathrm{Oe} / \mathrm{Oa}$ & $5-0$ & 176 & 496 & 12.180 & 1.284 & 98 & 786 & 1.210 & 8,4 & 84,0 & 3,2 \\
\hline & A & $0-6$ & 88 & 166 & 1.082 & 260 & 852 & 604 & 126 & 5,0 & 5,8 & 1,0 \\
\hline & B1 & $6-30$ & 62 & 48 & 76 & 62 & 2.382 & 640 & 4 & 5,8 & 0,2 & 0,2 \\
\hline & B2 & $30-60$ & 64 & 48 & 52 & 64 & 1.760 & 168 & 2 & 4,4 & 0,2 & 0,1 \\
\hline & B3 & $60-80$ & 76 & 62 & 178 & 192 & 994 & 160 & 2 & 3,6 & 0,2 & $<0,1$ \\
\hline 10 & $\mathrm{Oi} / \mathrm{Oe}$ & $10-4$ & 180 & 640 & 8.000 & 1.856 & 408 & 1.140 & 1.288 & 10,4 & 34,4 & - \\
\hline \multirow[t]{3}{*}{ Nothofagus pumilio } & $\mathrm{Oa}$ & $4-0$ & 124 & 204 & 3.200 & 668 & 894 & 986 & 488 & 6,0 & 12,2 & - \\
\hline & E & $0-7$ & 96 & 24 & 542 & 154 & 696 & 422 & 36 & 3,0 & 1,0 & $<0,1$ \\
\hline & $2 \mathrm{BC}$ & $7-16$ & 102 & 34 & 708 & 230 & 1.350 & 404 & 12 & 4,4 & 3,4 & $<0,1$ \\
\hline 11 & A & $0-5$ & 52 & 122 & 1.172 & 278 & 310 & 520 & 22 & 4,0 & 10,2 & 0,9 \\
\hline \multirow[t]{3}{*}{ Pradera } & E & $5-10$ & 26 & 116 & 280 & 128 & 264 & 160 & 2 & 3,0 & 2,2 & 0,6 \\
\hline & 2Bhs & $10-30$ & 34 & 126 & 256 & 134 & 2.744 & 1.370 & 2 & 6,4 & 0,2 & 0,8 \\
\hline & $\mathrm{BC}$ & $30-35$ & 26 & 56 & 114 & 52 & 5.216 & 1.120 & 2 & 4,6 & 0,2 & 0,3 \\
\hline
\end{tabular}

(-) No determinado 


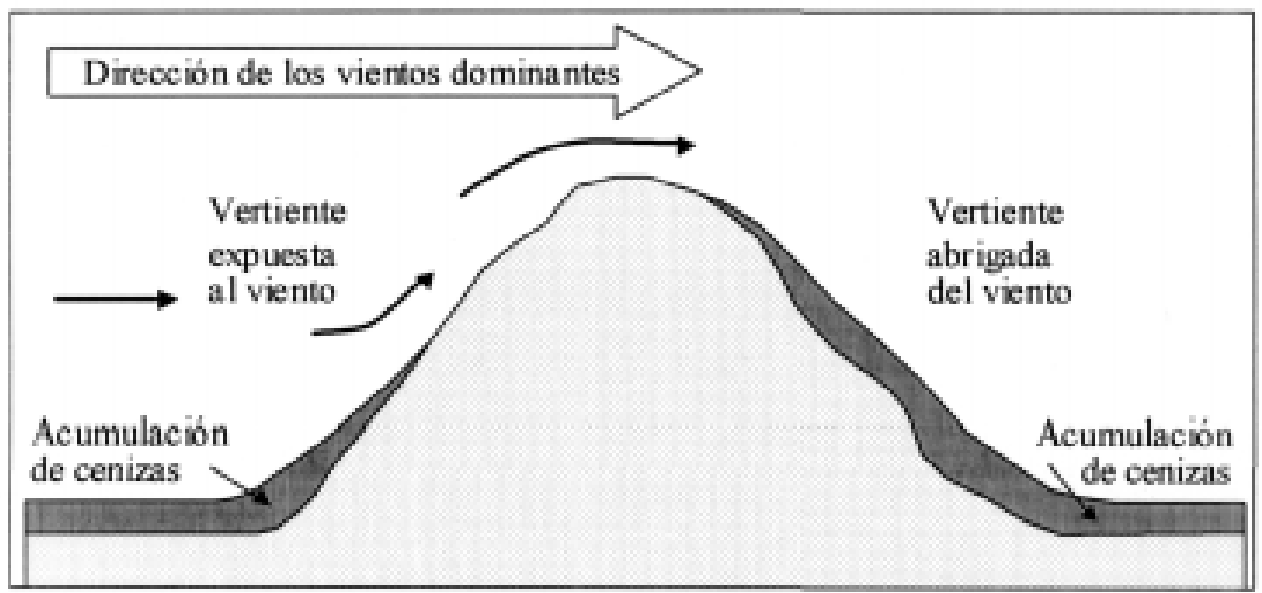

Fig. 4: Modelo de depósito de cenızas volcánıcas en la Patagonıa. Fuente: F. Colmet-Daage (recopilación inédita de datos).

Model of the deposition of volcanic ashes in Patagonia. Source: F. Colmet-Daage (compilation of unpublished data).

TABLA 7

Bases y aluminio de suelos en perfiles del sector Río Cóndor

Bases and aluminum in the soils in profiles of Río Cóndor

\begin{tabular}{|c|c|c|c|c|c|c|c|c|c|}
\hline $\begin{array}{l}\text { Perfil, tipo } \\
\text { de bosque }\end{array}$ & \multicolumn{2}{|c|}{$\begin{array}{c}\text { Horizonte Profundidad } \\
\qquad(\mathrm{cm})\end{array}$} & $\mathrm{Na}$ & $\mathrm{K}$ & \multicolumn{2}{|c|}{$\begin{array}{l}\mathrm{Ca} \quad \mathrm{Mg} \\
{\left[\mathrm{cmol}^{(+)} \mathrm{kg}^{-1}\right]}\end{array}$} & $\begin{array}{l}\text { Suma } \\
\text { de bases }\end{array}$ & $\begin{array}{c}\mathrm{Al} \\
\text { Intercam- } \\
\text { biable }\end{array}$ & $\begin{array}{c}\text { Saturación } \\
\text { de Al } \\
(\%)\end{array}$ \\
\hline 3 & $\mathrm{Oa}$ & 12 & 1,43 & 1,97 & 52,90 & 10,63 & 66,94 & - & . \\
\hline Nothofagus betuloides & A & $0-25$ & 0,60 & 0,53 & 4,60 & 0,86 & 6,59 & 5,16 & 43,9 \\
\hline \multirow[t]{2}{*}{ Nothofagus pumilio } & Bhs & $25-35$ & 0,24 & 0,19 & 1,30 & 0,60 & 2,33 & 9,67 & 80,6 \\
\hline & $\mathrm{C}$ & $35-45$ & 0,06 & 0,11 & 0,30 & 0,35 & 0,82 & 3,09 & 79,0 \\
\hline 4 & $\mathrm{ACg}$ & $0-8$ & 0,18 & 0,17 & 1,60 & 1,04 & 3,00 & 3,62 & 54,7 \\
\hline Nothofagus betuloides & $\mathrm{C}$ & $8-20$ & 0,20 & 0,17 & 5,10 & 2,98 & 8,44 & 7,67 & 47,6 \\
\hline 5 & A & $0-6$ & 0,59 & 0,20 & 1,00 & 1,26 & 3,05 & 5,20 & 63,1 \\
\hline Nothofagus betuloides & B & $6-30$ & 0,52 & 0,09 & 0,57 & 0,55 & 1,72 & 8,16 & 82,5 \\
\hline Nothofagus pumilio & $\mathrm{C}$ & $30-32$ & 0,22 & 0,09 & 0,74 & 0,58 & 1,62 & 11,44 & 87,6 \\
\hline 6 & A & $0-20$ & 0,48 & 0,15 & 1,09 & 0,76 & 2,48 & 9,98 & 80,1 \\
\hline Nothofagus betuloides & $\mathrm{C}$ & $20-40$ & 0,31 & 0,13 & 4,78 & 1,47 & 6,69 & 1,76 & 20,8 \\
\hline 7 & $\mathrm{Oi}$ & $7-5$ & 0,76 & 1,66 & 54,40 & 12,89 & 69,71 & - & - \\
\hline \multirow[t]{5}{*}{ Nothofagus pumilio } & $\mathrm{Oe} / \mathrm{Oa}$ & $5-0$ & 0,77 & 1,27 & 60,90 & 10,61 & 73,55 & 0,73 & 1,0 \\
\hline & A & $0-6$ & 0,38 & 0,42 & 5,41 & 2,15 & 8,37 & 5,98 & 41,7 \\
\hline & B1 & $6-30$ & 0,27 & 0,12 & 0,38 & 0,51 & 1,28 & 10,51 & 89,1 \\
\hline & B2 & $30-60$ & 0,28 & 0,12 & 0,26 & 0,53 & 1,19 & 10,89 & 90,1 \\
\hline & B3 & $60-80$ & 0,33 & 0,16 & 0,89 & 1,59 & 2,97 & 8,91 & 75,0 \\
\hline 10 & $\mathrm{Oi} / \mathrm{Oe}$ & $10-4$ & 0,78 & 1,64 & 40,00 & 15,34 & 57,76 & - & - \\
\hline \multirow[t]{3}{*}{ Nothofagus pumilio } & $\mathrm{Oa}$ & $4-0$ & 0,54 & 0,52 & 16,00 & 5,52 & 22,58 & - & 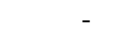 \\
\hline & $\mathrm{E}$ & $0-7$ & 0,42 & 0,06 & 2,71 & 1,27 & 4,46 & 4,58 & 50,6 \\
\hline & $2 \mathrm{BC}$ & $7-16$ & 0,44 & 0,09 & 3,54 & 1,90 & 5,97 & 5,78 & 49,2 \\
\hline 11 & A & $0-5$ & 0,23 & 0,31 & 5,86 & 2,30 & 8,70 & 1,24 & 12,5 \\
\hline \multirow[t]{3}{*}{ Pradera } & $\mathrm{E}$ & $5-10$ & 0,11 & 0,30 & 1,40 & 1,06 & 2,87 & 3,98 & 58,1 \\
\hline & 2Bhs & $10-30$ & 0,15 & 0,32 & 1,28 & 1,11 & 2,86 & 6,71 & 70,1 \\
\hline & $\mathrm{BC}$ & $30-35$ & 0,11 & 0,14 & 0,57 & 0,43 & 1,26 & 5,87 & 82,4 \\
\hline
\end{tabular}

(-) No determinado 
por presentar un horizonte cámbico y desarrollarse en zonas con bajas temperaturas.

En concordancia con lo anterior, estos suelos tienen en general una fuerte correspondencia con los suelos pardo podzólicos o pardo forestales descritos por Díaz et al. (1960) y Pisano (1977), porque se trataría de suelos jóvenes con influencia volcánica.

\section{Caracterización química de los suelos}

En el sector Río Bueno el mantillo (horizontes Oi, Oe y $\mathrm{Oa}$ ) (Tabla 2) presentó un pH muy fuertemente ácido a fuertemente ácido $(=4,8-5,5)$, contenidos de nitrógeno total muy altos $(0,4-0,9 \%)$, una relación $\mathrm{C} / \mathrm{N}$ de $15-20$ y una concentración de fósforo de 1-52 $\mathrm{mg} \mathrm{kg}^{-1}$. El potasio presentó un nivel de 206-1.776 $\mathrm{mg} \mathrm{kg}^{-1}$, el calcio de 1.810$10.280 \mathrm{mg} \mathrm{kg}^{-1}$ y el magnesio de 698-1.914 mg $\mathrm{kg}^{-1}$ (Tabla 3); el sodio, en cambio, presentó niveles menores (108-472 $\mathrm{mg} \mathrm{kg}^{-1}$ ). De acuerdo con los niveles de referencia propuestos por Rodríguez (1992), los elementos hierro, manganeso, zinc y cobre presentaron contenidos muy altos y el boro un nivel alto (Tabla 3); el aluminio extraible (Tabla 3) e intercambiable (Tabla 4) presentaron niveles bajos, al igual que la saturación de aluminio. En algunos casos los niveles de aluminio fueron altos, lo que se explica por la presencia de suelo mineral entremezclado con el material orgánico del mantillo (horizontes $\mathrm{Oa}$ ). La suma de bases fue muy alta, dominada por el calcio, luego magnesio y en menor proporción potasio y sodio. Estos dos últimos cationes son de mayor solubilidad y es frecuente su pérdida por lixiviación en sustratos bajo climas húmedos.

El suelo mineral del sector Río Bueno (Tabla 2) también presentó un pH ácido $(=4,1-5,4)$, generalmente más ácido que el mantillo. Los contenidos de carbono total $(0,7-11,3 \%)$ y nitrógeno total $(0,09-0,66 \%)$ presentaron amplios rangos, en cambio la relación $\mathrm{C} / \mathrm{N}$ fue baja a media (2 - 39). El fósforo (Tabla 2) y las bases (Tabla 3 y 4) mostraron niveles mucho menores que los encontrados en el mantillo. En general estos elementos, según Rodríguez (1992), varían en niveles bajos a muy altos, con una disminución de las concentraciones con la profundidad. Los elementos menores presentaron contenidos muy variables (Tabla 3). El aluminio extraible (Tabla 3 ) e intercambiable y la saturación de aluminio (Tabla 4) presentaron niveles mucho mayores que en el mantillo, alcanzando una categoría de muy altos, por lo que el suelo mineral presentaría condiciones químicas restrictivas para el desarrollo radicular debido a condiciones de toxicidad (Rodríguez 1992).
En el sector Río Cóndor el mantillo presentó características químico-nutritivas similares a las encontradas en Río Bueno (Tabla 5, 6 y 7). Es decir, pH ácido, altos contenidos de nitrógeno total y una relación $\mathrm{C} / \mathrm{N}$ baja. El fósforo disponible y las bases presentaron niveles altos. Los elementos menores hierro, manganeso y zinc presentaron niveles muy altos, en cambio para el cobre fueron altos. No se determinó boro, aluminio intercambiable ni saturación de aluminio.

En el suelo mineral en el sector Río Cóndor (Tabla 5, 6 y 7) las condiciones nutritivas resultaron menos favorables que en Río Bueno, es decir, el pH fue más ácido, el fósforo y las bases presentaron menores concentraciones y el aluminio alcanzó niveles mayores. Sin embargo, la materia orgánica, el nitrógeno total y los elementos menores mostraron niveles similares a los de Río Bueno.

En los perfiles de suelo del sector de Vicuña (Tabla 2, 3 y 4) se encontraron características químicas dentro de los rangos observados en Río Bueno.

\section{Arraigamiento}

El arraigamiento se concentró principalmente en el mantillo y disminuyó fuertemente con la profundidad, siguiendo la misma tendencia de los elementos nutritivos e inversa a la saturación de aluminio (Tabla 4 y 7). Esta distribución de las raíces también se relaciona con la estructura suelta del mantillo, lo que le permite un buen desarro1lo radicular y mayor retención de agua que en los niveles más profundos.

La elevada pedregosidad a partir de los 20-30 cm de profundidad limitaría también la distribución de las raíces. Debido a que el desarrollo radicular se concentró en la parte superior del perfil, las raíces se desarrollaron lateralmente en mayor proporción que en profundidad. Este tipo de desarrollo radicular sumado a las características climáticas de nieve y fuertes vientos típicos de esta zona (Pisano 1977), son factores que afectan la susceptibilidad del dosel a las perturbaciones y se deben considerar al momento de intervenir silviculturalmente el bosque. La caída de árboles por desarraigamiento es frecuente en toda la zona de estudio.

\section{Fertilidad del suelo y factores limitantes}

La fertilidad del suelo incluye al espacio arraigable y los regímenes de agua, aire, calor y de elementos nutritivos. De acuerdo a estos factores, los suelos en que se desarrolla coigüe de Magallanes 
y especies asociadas en Tierra del Fuego presentan una baja fertilidad, en atención a los factores limitantes que se señalan a continuación.

(1) Escasa profundidad arraigable del suelo, especialmente en lugares donde la elevación supera los $200 \mathrm{~m}$ y la pendiente es mayor a $20 \%$. Esto se ve agravado por altos volúmenes de pedregosidad en los suelos $(>30 \%)$ en la parte media y más profunda del perfil. Como consecuencia, la capacidad de agua aprovechable es baja.

(2) Drenaje interno lento en los terrenos planos o de escasa pendiente, lo que limita la aireación del suelo.

(3) Las condiciones químico-nutritivas del suelo mineral son desfavorables, en particular se encuentra alta acidez, baja disponibilidad de elementos nutritivos y altos niveles de aluminio. La existencia del horizonte E restringe el desarrollo de las raíces a mayor profundidad, posiblemente por su elevada acidez y sus altos niveles de aluminio. En todo caso, los niveles de acidez observados están dentro de los rangos normales para suelos bajo bosques nativos de estas latitudes australes (Sadzawka et al. 1995).

Un componente esencial de la fertilidad lo constituye el mantillo, dadas sus características adecuadas para un intenso arraigamiento y elevada oferta de elementos nutritivos. Sin embargo, su escasa profundidad para sostener vegetación arbórea representa una fragilidad que debe ser considerada al momento de intervenir silviculturalmente el bosque, para mantener la calidad de los sitios (Donoso et al. $1995^{9}$, Thiers 1997).

\section{CONCLUSIONES}

Los suelos bajo coigüe de Magallanes y especies asociadas en Tierra del Fuego son jóvenes, poco evolucionados, fuertemente influidos por la actividad volcánica de la Región. La mayor parte de los suelos muestreados pertenecen a los órdenes Spodosol e Inceptisol. El primer tipo se desarrolla frecuentemente en sectores planos, a baja elevación, en tanto que los segundos se ubican generalmente en toda la topografía.

En general, el suelo mineral es delgado $(<50$ $\mathrm{cm})$, con alto volumen de pedregosidad (10-70\%) que aumenta en profundidad, de textura franca, estructura masiva a granular, capacidad de agua aprovechable baja (<60 $\mathrm{mm})$ y drenaje externo e interno moderado a lento. Son fuertemente ácidos y con una oferta nutricional pobre de los elementos mayores (nitrógeno, fósforo, potasio, magnesio). Destacan además, la elevada concentración de aluminio (> $30 \%$ saturación de aluminio) en las capas profundas.

En todos los bosques se presenta mantillo con un espesor más frecuente de $4-8 \mathrm{~cm}$, donde la forma de humus es del tipo moder a mor. Las raíces de árboles se concentran en esta capa orgánica, debido principalmente a las altas concentraciones de nutrientes y a las condiciones físicas que le dan al mantillo una estructura suelta. Por lo anterior, la nutrición de los bosques de esta zona depende fuertemente de la conservación de la delgada capa de mantillo.

Frecuentemente, en toda la zona de estudio, debajo del mantillo se encuentra un horizonte de color blanco (comúnmente de color 10 YR 5/1-2), de textura franco limosa y estructura masiva. Sus características químicas son desfavorables para el arraigamiento. Presenta una alta acidez, bajos niveles de nutrientes y niveles tóxicos de aluminio.

Respecto al origen de este horizonte blanco se plantea como hipótesis que, por sus características morfológicas y químicas, y por su frecuencia y ubicación en la topografía, se trataría de una capa distinta al suelo subyacente, originada de cenizas volcánicas de carácter ácido, depositadas por el viento y afectada a través de los años por procesos de eluviación, generando suelos del orden Spodosol. Su ausencia en sectores más expuestos se explicaría por efecto de erosión eólica.

La fertilidad de los suelos bajo coigüe de Magallanes y especies asociadas es en general baja. Entre los principales factores limitantes se encuentran el reducido espacio arraigable, la baja capacidad de agua aprovechable y la baja disponibilidad de elementos nutritivos en las capas profundas; por otra parte, se encuentran altos contenidos de aluminio con características tóxicas para las raíces.

\section{AGRADECIMIENTOS}

Los autores agradecen el apoyo brindado por la empresa Trillium Ltda. para la realización de este trabajo. De igual manera a los profesores M.Sc. Walter Luzio (Facultad de Ciencias Agrarias y Forestales, Universidad de Chile) y Dr. Juan E. Schlatter (Facultad de Ciencias Forestales, Universidad Austral de Chile) por sus comentarios que contribuyeron a mejorar este trabajo.

${ }^{9}$ Op. cit. 


\section{LITERATURA CITADA}

CORPORACIÓN NACIONAL FORESTAL (CONAF), COMISIÓN NACIONAL DEL MEDIO AMBIENTE (CONAMA) \& BANCO MUNDIAL (BIRF) (1997)

Catastro y evaluación de los recursos vegetacionales nativos de Chile. Informe Nacional con Variables Ambientales. Comisión Nacional del Medio Ambiente, Santiago, Chile. 78 pp.

DÍAZ C, C AVILÉS \& R RAY (1960) Los grandes grupos de suelos de la provincia de Magallanes. Ministerio de Agricultura, Agricultura Técnica (Chile) 18: 227 308.

DOLLENZ O (1995) Los árboles y bosques de Magallanes. Ediciones de la Universidad de Magallanes, Punta Arenas, Chile. 123 pp.

DONOSO C (1981) Tipos forestales de los bosques nativos de Chile. Documento de Trabajo No. 38. Investigación y Desarrollo Forestal (CONAF-PNUD-FAO), Santiago, Chile. 70 pp.

FUENZALIDA H (1965) Geografía económica de Chile: clima. Corporación de Fomento de la Producción, Santiago, Chile. 885 pp.

GONZÁLEZ-FERRÁN O (1995) Volcanes de Chile. Instituto Geográfico Militar, Santiago, Chile. 640 pp.

HARTUNG J, B ELPELT \& K-H KLÖSENER (2002) Statistik. Lehr- und Handbuch der angewandten Statistik. Oldenbourg Verlag, München, Germany. $975 \mathrm{pp}$.

INTA-ORSTOM (1991) Suelos con aluminio activo, montmorillonita, clorita, ilita, vermiculita interestratificaciones regular o irregularmente. Universidad Nacional del Comahue, Facultad de Ciencias Agrarias, Río Negro, Argentina. 133 pp.
INSTITUTO DE RECURSOS NATURALES (IREN) (1967) Provincia de Magallanes: inventario preliminar de los recursos naturales. Zona continental e isla de Tierra del Fuego. Santiago, Chile. (Mimeo.) 151 pp.

PISANO E (1977) Fitogeografía de Fuego-Patagonia Chilena. I. Comunidades vegetales entre las latitudes 51 y $56^{\circ}$ Sur. Punta Arenas, Chile. Anales del Instituto de la Patagonia (Chile) 8: 121-250.

RODRÍGUEZ J (1992) Manual de fertilización. Colección en Agricultura, Facultad de Agronomía, Ediciones de 1a Pontificia Universidad Católica de Chile, Santiago, Chile. 362 pp.

SADZAWKA A, M PERALTA, M IBARRA, J PERALTA \& J FUENTES (1995) Características químicas de los suelos forestales chilenos. Bosque (Chile) 16: 9-28.

SOIL CONSERVATION SERVICE (1983) Keys to soil taxonomy. Soil Management Support Service, New York, Technical Monograph No. 6. 244 pp.

THIERS O (1997) Estudio de relaciones entre los bosques de coigüe de Magallanes (Nothofagus betuloides (Mirb) Blume) y el suelo en Tierra del Fuego. Tesis de Ingeniería Forestal, Facultad de Ciencias Forestales, Universidad Austral de Chile, Valdivia, Chile. $101 \mathrm{pp}$.

UNITED STATED DEPARTMENT OF AGRICULTURE (1999) Soil taxonomy: a basic system of soil classification for making and interpreting soil surveys. Seconds edition. Agriculture Handbook No. 436, Washington, District of Columbia. 870 pp. 